\title{
血液相容性聚氨酯弹性体的合成与性能研究
}

\author{
鞠明杰 $a$ 徐涁涁 $*, b$ 徐立功 $*, a$ \\ ( ${ }^{a}$ 华东理工大学体育科学与工程学院 上海 200237) \\ ( ${ }^{b}$ 华东理工大学材料科学与工程学院 上海 200237)
}

\begin{abstract}
摘要 聚氨酯材料被广泛用于植入导管及药物载体等生物医用领域, 但在一些使用环境中，其血液相容性仍然不能达 到要求. 以聚四氢呋喃醚二元醇(PTMG)、聚碳酸酯二元醇(PC)为软段, 异佛尔酮二异氭酸酯(IPDI)为硬段, 1,4-丁二醇 (BDO)、二甲硫基甲苯二胺(E300)为扩链剂, 设计合成了具有良好血液相容性的聚氨酯弹性体. 对合成的聚氨酯弹性体 进行红外光谱(FT-IR)、差示扫描量热分析(DSC)、力学性能和水接触角的测试, 并进行了溶血实验和细胞相容性实验. 结果表明, 所合成的聚氨酯弹性体具有良好的机械力学性能; 溶血和细胞相容性实验显示该材料具有优良的血液相容 性. 特别是选用 IPDI 和 E-300 扩链剂作硬段、PC 作软段的聚氨酯弹性体的综合性能最佳，在生物医疗等领域具有一定 的应用前景.
\end{abstract}

关键词 聚氨酯; 弹性体; 溶血实验; 血液相容性

\section{Synthesis and Properties of Blood Compatible Polyurethane Elastomer}

\author{
$\mathrm{Ju}$, Mingjie ${ }^{a} \quad \mathrm{Xu}$, Binbin $^{*, b} \quad \mathrm{Xu}$, Ligong ${ }^{*, a}$ \\ ( ${ }^{a}$ School of Sports Science and Engineering, East China University of Science and Technology, Shanghai 200237) \\ ( ${ }^{b}$ School of Materials Science and Engineering, East China University of Science and Technology, Shanghai 200237)
}

\begin{abstract}
Polyurethane materials are widely used in biomedical fields such as implantable catheters and drug delivery. However, their blood compatibility is difficult to meet the rigorous requirements needed for a new generation of biomedical devices. A series of polyurethane elastomers were prepared using polycarbonate diols (PC), polyoxytertramethylene glycol (PTMG), isophorone diisocyanate (IPDI) and chain extender (BDO, E-300) as raw materials. The properties of polyurethane elastomers were investigated by FT-IR spectrum, differential scanning calorimeter (DSC) analysis, mechanical test, water contact angle measurement, hemolysis and cell compatibility test. The mechanical results show that the materials have superior tensile strength and elongation, meanwhile, the homolysis and cells compatibility tests exhibit that they have good biocompatibility. In addition, the materials can achieve the best performance by using IPDI as well as chain extender E-300 as the hard segment and PC as the soft segment.
\end{abstract}

Keywords polyurethane; elastomer; hemolysis; blood compatibility

过去几十年中，聚氨酯(PU)弹性体材料取得了广泛 的应用，其可以在微观结构上发生软硬段分离而具备比 其它材料更好的血液相容性 ${ }^{[1-3]}$. 同时 PU 弹性体还具有 优异的弹性和强度, 因此各类聚氨酯材料被广泛用于植 入导管、器械涂层及药物载体等生物医用领域 $[4-8]$. 虽然 PU 材料在生物医用领域的应用已经接近 40 年, 但在一 些使用环境中, 其血液相容性仍然不能达到要求. 因此, 改善 PU 材料的血液相容性得到了科研工作者的广泛关
注 ${ }^{[9-12]}$. 常用的介入导管医用 PU 材料以聚四氢呋喃醚 二元醇 (PTMG) 为软段, 先与 4,4'-二苯基二异氧酸酯 (MDI)反应生成预聚物，再使用小分子二醇或二胺为扩 链剂来合成. 为了明确不同的软段和小分子扩链剂对 $\mathrm{PU}$ 弹性体材料力学性能和血液相容性的影响, 本工作 以异佛尔酮二异氧酸酯(IPDI)为硬段、PTMG 和聚碳酸 酯二元醇(PC)为软段、1,4-丁二醇(BDO)或二甲硫基甲苯 二胺(E300)为扩链剂制备了一系列样品, 并对其进行了

* Corresponding authors. E-mail: binbinxu@ecust.edu.cn; xuligong@ecust.edu.cn

Received June 4, 2020; revised June 16, 2020; published online August 5, 2020.

Project supported by National Natural Science Foundation of China (No. 22001072), and Shanghai Sailing Program (No. 19YF1410300)

国家自然科学基金(No. 22001072)和上海市青年科技英才扬帆计划(No. 19YF1410300)资助项目. 
性能表征.

\section{1 结果与讨论}

\section{1 聚氨酯弹性体材料的合成与红外光谱(FT-IR)结构 分析}

合成聚氨酷弹性体的路线示意图见 Scheme 1. 首先 选用 IPDI 作为硬端骨架引入异氰酸酯活性位点, 分别 利用 PTMG 或者 PC 为活性二醇位点, 在二月桂酸二丁 基锡的催化下 $80{ }^{\circ} \mathrm{C}$ 反应 $3 \mathrm{~h}$, 制备聚醚型或者聚酯型聚 氨酯预聚物(Scheme 1, A). 随后加入 E300 作为氨类扩 链剂或者 BDO 作为醇类扩链剂, 固化形成最终的聚氨 酯弹性体材料(Scheme 1, B). 选用不同的软段二醇结构 以及不同的氨类或者醇类扩链剂, 合成了四种不同种类 的聚氨酯弹性体(IPDI-PTMG-E300、IPDI-PTMG- BDO、 IPDI-PC-E300 和 IPDI-PC-BDO), 如表 1 所示. 不同种类 聚氨酯的硬段比例都控制在 $38 \%$ 左右, 以便进行后续性 能之间的对比.

表 1 聚氨酯弹性体的配方组成及硬段含量

Table 1 Composition of different polyurethane elastomers

\begin{tabular}{ccc}
\hline Sample & Composition & Hard segment content $/ \%$ \\
\hline $\mathbf{1}$ & IPDI-PTMG-E300 & 37.00 \\
$\mathbf{2}$ & IPDI-PTMG-BDO & 38.09 \\
$\mathbf{3}$ & IPDI-PC-E300 & 37.00 \\
$\mathbf{4}$ & IPDI-PC-BDO & 38.09 \\
\hline
\end{tabular}

图 1 是所合成的聚氨酯弹性体的典型的红外光谱曲
线, 样品 1 在 2973, 2833 和 $1109 \mathrm{~cm}^{-1}$ 处的吸收峰证明 了样品的聚梄型聚氨酯结构, 样品 $\mathbf{3}$ 在 1726 和 1264 $\mathrm{cm}^{-1}$ 处的吸收峰证明了所合成的聚酯型聚氨酯的结构. 图中的两条光谱曲线在 $3700 \mathrm{~cm}^{-1}$ 附近存在极其微弱的 $\mathrm{OH}$ 吸收峰，在 $2300 \mathrm{~cm}^{-1}$ 附近没有明显的 $\mathrm{NCO}$ 吸收峰, 说明样品中几乎没有残余的反应官能团, 整个合成反应 进行的较为完全.

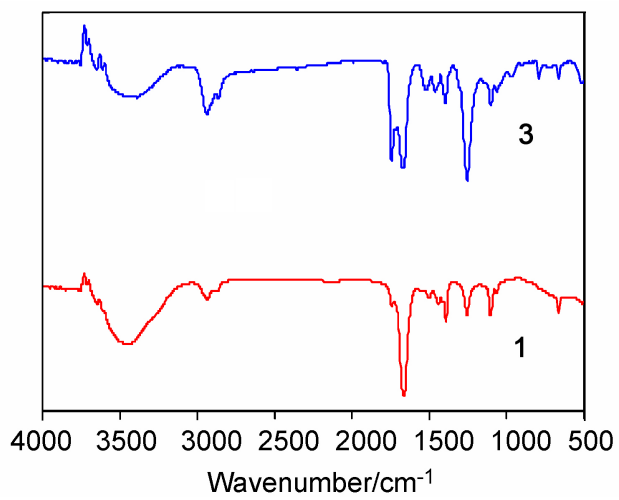

图 1 聚氨酯样品 $\mathbf{1}$ 和 $\mathbf{3}$ 的红外光谱图

Figure 1 FT-IR spectra of samples 1 and $\mathbf{3}$.

\section{2 亲水性分析}

生物体内多种体液包括血液均为含水体系, 因此, 生物材料的亲水性对其血液相容性十分重要. 聚氨酯的 亲水性可以通过表面的水接触角进行衡量, 水接触角反 映表面被湿润的难易程度 ${ }^{[13]}$. 水接触角越小, 材料表面<smiles>CC1(C)CC([N+](=O)[O-])CC(C)(CNC(=O)OOC(=O)NCC2(C)CC([N+](=O)[O-])CC(C)(C)C2)C1</smiles>

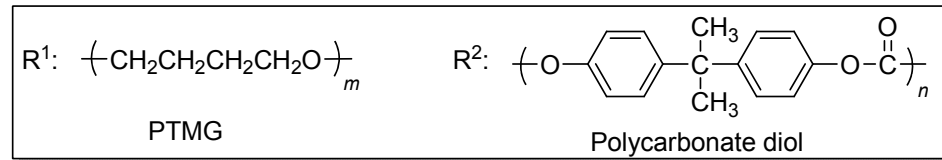

(B)<smiles>CCOC(=O)NCC1(C)CC(NC(=O)OCCCCOC(=O)NC2CC(C)(C)CC(C)(C)C2)CC(C)(C)C1</smiles>

Pre-polyme

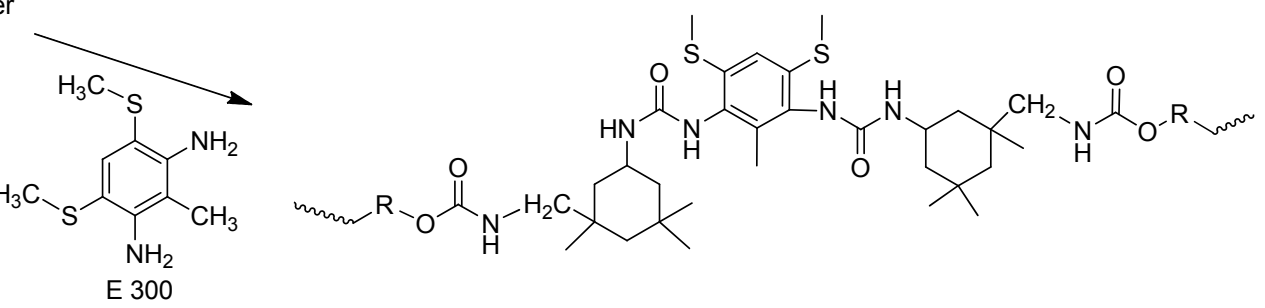

图式 1 不同种类聚氨酯弹性体的合成

Scheme 1 Synthesis of different polyurethane elastomers 
亲水性越好. 表 2 中为合成的部分聚氨酯弹性体的水接 触角测试结果.

表 2 不同体系聚氨酯弹性体的水接触角与力学性能 Table 2 Water contact angles and mechanical properties of different polyurethane elastomers

\begin{tabular}{cccc}
\hline Sample & $\begin{array}{c}\text { Water contact } \\
\text { angle }\end{array}$ & Strength/\% & $\begin{array}{c}\text { Elongation/\% } \\
\text { at break }\end{array}$ \\
\hline $\mathbf{1}$ & $52.7^{\circ}$ & 12.5 & 249.7 \\
$\mathbf{2}$ & $68.1^{\circ}$ & 8.8 & 348.5 \\
$\mathbf{3}$ & $46.2^{\circ}$ & 16.8 & 226.4 \\
$\mathbf{4}$ & $58.6^{\circ}$ & 10.8 & 257.0 \\
\hline
\end{tabular}

从表 2 中的接触角数据可以看出, 样品 $\mathbf{1}$ 的水接触 角 $\left(52.7^{\circ}\right)$ 明显小于样品 $2\left(68.1^{\circ}\right)$, 其二者在配方上只有 扩链剂种类的差异, 说明氨类扩链剂的样品与水之间的 相容性更好, 这是由于相较于醇类扩链剂形成的氨基甲 酸酯键, 氨类扩链剂会在聚合物分子链上形成大量艮 基, 而脲基与水能形成更多的氢键. 同样的结论在样品 3 和 4 的测试结果 $\left(46.2^{\circ}\right.$ vs. $\left.58.6^{\circ}\right)$ 上也得到验证. 同时, 样品 1 的水接触角 $\left(52.7^{\circ}\right)$ 明显大于样品 $3\left(46.2^{\circ}\right)$, 说明 聚酯结构较聚醚结构更为亲水.

\section{3 力学性能}

从表 2 中不同体系聚氨酯的力学性能数据可以看 出, 4 个样品在硬段含量基本相同的情况下, 采用聚酯 多元醇的力学强度高于聚醚多元醇, 如样品 $\mathbf{3}$ 的强度 (16.8\%)大于对应的聚醚样品 1 的强度(12.5\%), 样品 $\mathbf{4}$ 的强度 $(10.8 \%)$ 大于对应的聚醚样品 $\mathbf{2}$ 的强度 $(8.8 \%)$; 采 用氨类扩链剂的样品强度高于醇类扩链剂样品, 如样品 1 的强度(12.5\%)大于对应的聚醚样品 2 的强度 $(8.8 \%)$, 样品 3 的强度(16.8\%)大于对应的聚醚样品 4 的强度 $(10.8 \%)$; 而断裂伸长率则与此相反, 采用聚酯多元醇的 断裂伸长率低于聚醚多元醇, 如样品 $\mathbf{3}$ 的断裂伸长率 (226.4\%)小于对应的聚醚样品 $\mathbf{1}$ 的断裂伸长率(249.7\%), 样品 4 的断裂伸长率 $(257.0 \%$ )小于对应的聚醚样品 $\mathbf{2}$ 的 断裂伸长率 $(348.5 \%$ ); 采用氨类扩链剂的样品断裂伸长 率低于醇类扩链剂样品, 样品 1 的断裂伸长率 $(249.7 \%)$ 小于对应的聚醚样品 $\mathbf{2}$ 的断裂伸长率(348.5\%), 样品 $\mathbf{3}$ 的断裂伸长率 $(226.4 \%$ )小于对应的聚醚样品 $\mathbf{4}$ 的断裂伸 长率 $(257.0 \%)$. 为了更好地理解 PU 样品力学性能与内 部微观形貌之间的关系, 利用扫描电镜对不同的样品 $\mathbf{2}$ 和 3 进行分析(图 2).

由图 2 可观察到两个样品均为明暗相间的形态结 构 ${ }^{[14]}$, 其中凸出的较亮区域为硬段相, 较暗区域为刻蚀 掉的软段相, 表明聚氨酯嘱呈现明显的微观分相结构. 比较图 2 可见, 样品 2 采用 $\mathrm{PTMG}$ 和 $\mathrm{BDO}$ 作为原料, 硬 段间未形成较强的氢键, 此时软段为连续相, 硬段粒子 不均匀分散在软段中, 且数量较少; 样品 3 采用 PC 和
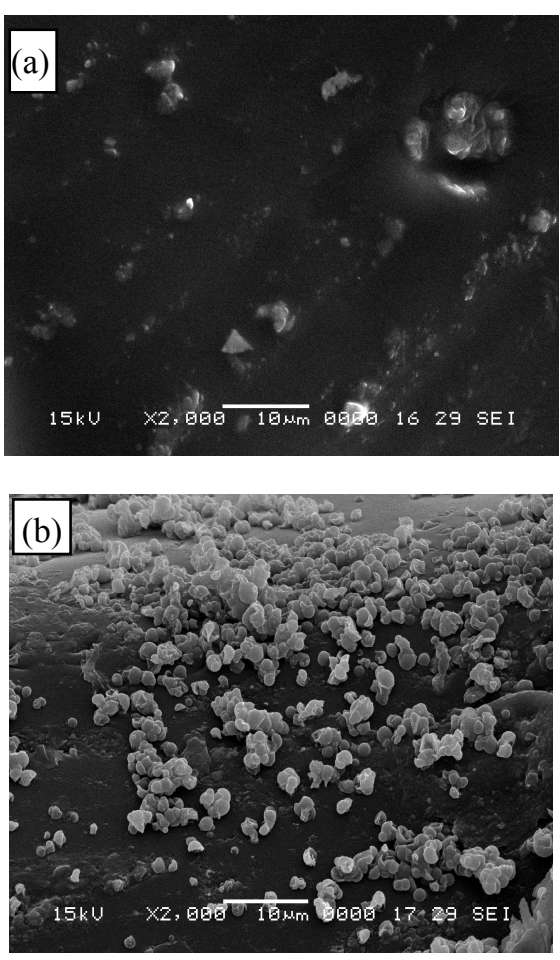

图 2 样品 2 (a) 和 3 (b)的扫描电子显微镜(SEM) 图 Figure 2 Scanning electron microscope (SEM) images of samples $\mathbf{2}$ (a) and $\mathbf{3}$ (b)

E300 为原料, 硬段形成氢键程度高, 此时软段虽仍然为 连续相，但硬段间已形成均匀粒子，同时数量明显增多， 在软段上的分布也更连续. 这种微观结构的不同导致样 品 3 有更高的强度，也会一定程度地影响其断裂伸长率, 可以很好地解释表 2 中力学性能的测试结果.

为了验证硬段间氢键对微观聚集结构的影响, 对样 品 2 和 3 的红外谱图羰基区进行分峰以明确其氢键化比 例. 两个样品羰基区红外光谱如图 3 所示, 两个样品的 峰形较宽, 推测是游离羰基和氢键化羰基峰的多重重叠 所引起. 为此采用自卷积最小二乘法 ${ }^{[15]}$ 对羰基区谱带 进行分峰拟合. 聚氨酯样品红外谱图中, 羰基区峰的归 属可以根据氢键化程度简单分为: 在 $1660 \sim 1740 \mathrm{~cm}^{-1}$ 范围内，低波数区域属于氢键的有序峰(ordered hydrogen bonding), 中波数区域属于氢键的无序峰(disordered hydrogen bonding), 高波数区域属于无氢键约束的羰基 峰(free).

$$
X=\frac{\text { Area }(\text { ordered }+ \text { disordered })}{\text { Total area }}
$$

最终的分峰结果如图 4 和表 3 所示, 从中可以看出 样品 2 的氢键化程度为 0.78 , 而有序化氢键化程度为 0.26 , 而样品 3 硬段的氢键化程度为 0.83 , 有序化氢键 程度为 0.32 , 可见样品 $\mathbf{3}$ 氢键化程度较高, 且有序氢键 的键能也高. 这是由于样品 3 采用 PC 和 $\mathrm{E} 300$ 为原料, 


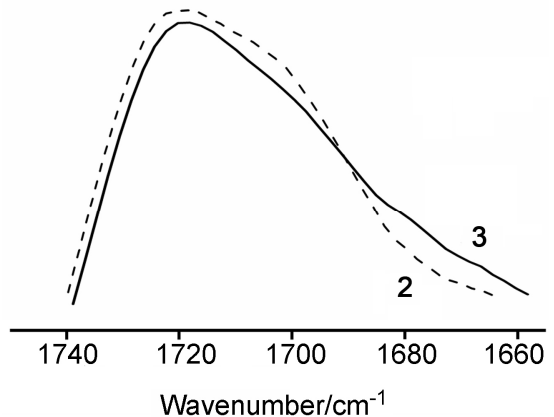

图 3 样品 2 和 3 的羰基区红外光谱

Figure 3 Carbonyl absorption of samples 2 and 3 in FT-IR spectra

赋予了硬段更高的氢键程度, 硬段间形成均匀粒子, 在 软段上可以更连续地分布. 这与力学性能测试结果及 SEM 测试结果相符合.

\section{4 细胞相容性分析}

从图 5 可以看出, 本实验所合成的聚氨醌弹性体表 现出了极好的细胞相容性, 从软段上看本实验选用的聚 醚型二元醇和聚酯型二元醇都有一定的极性, 易与细胞 培养液中的水形成氢键，从而提高材料表面的氧含量. 由于聚酯型二元醇本身的氧含量比聚醚型要高, 因此其 细胞生长率高于聚醚型聚氨酯. 从扩链剂上看, 本实验 主要采用了二醇类的 $\mathrm{BDO}$ 和二胺类的 $\mathrm{E} 300$, 采用 $\mathrm{E} 300$ 扩链的聚合物的细胞生长情况明显好于 $\mathrm{BDO}$, 这是由 于用 E300 来扩链, 材料表面引入了更多的含氮基团, 不仅可以使材料表面带上一定的正电荷(胺的阳离子 化), 以调节材料表面的亲疏水性, 而且可以和细胞蛋

(a)

Disordered $\mathrm{H}$-bonded

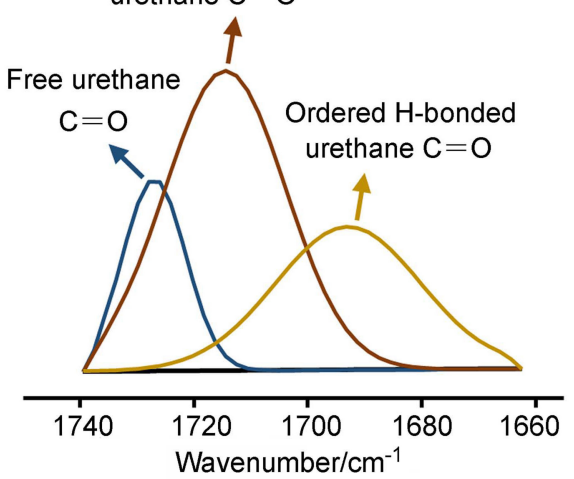

白质肽链发生官能团之间的作用，从多角度促进细胞的 生长 ${ }^{[16-18]}$.

\section{5 血液相容性}

溶血实验是利用生物材料或其浸提液进行体外实 验, 测定红细胞溶解和血红蛋白游离的程度, 从而对生 物医用材料或其制品的体外溶血性进行评价 ${ }^{[19-21]}$. 从表 4 中可以看出 4 个样品溶血率均小于 $5 \%$, 基本保持在 $3 \%$ 以下, 说明材料对血红细胞的破坏很小, 因此本实 验所合成的聚氨酯弹性体可以满足生物材料的溶血性 要求.

\section{2 结论}

选用 IPDI、不同的软段二醇结构以及不同的氨类或者醇 类扩链剂, 合成了四种不同种类的聚氨酯弹性体 IPDI-PTMG-E300、IPDI-PTMG-BDO 、IPDI-PC-E300 和 IPDI-PC-BDO. 对比所合成的一系列聚氨酯样品的 力学性能, 选用聚酯型的多元醇为软段和 E300 为扩链 剂合成所得聚氨酯弹性体性能要优于聚醚型软段和 $\mathrm{BDO}$ 为扩链剂的聚氨酯弹性体. 由于 $\mathrm{E} 300$ 和 PC 分子 中含有亲水基团, 以此为扩链剂和软段所合成的聚氨酯 弹性体形成氢键程度较高, 具有较好的亲水性和良好的 血液相容性. 因此, 所设计聚酯型聚氨酯以其优异的力 学性能与血液相容性在生物医疗领域具有一定的应用 前景, 在后续深入研究中将进一步开展聚酯型聚氨酯的 成型加工应用，建立不同的功能性模块，更加全面地衡 量聚酯型聚氨酯的生物应用价值.

(b)

Disordered $\mathrm{H}$-bonded urethane $\mathrm{C}=\mathrm{O}$

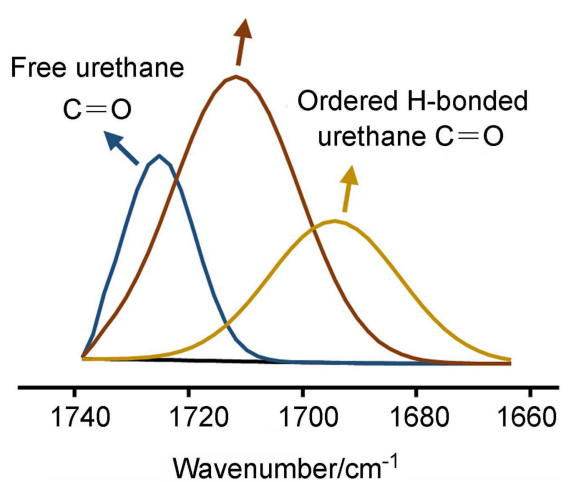

图 4 样品 $\mathbf{2}$ (a) 和 $\mathbf{3}$ (b) 红外羰基区的红外分峰结果

Figure 4 Differentiation of $\mathrm{C}=\mathrm{O}$ stretching of samples 2 (a) and 3 (b) in FT-IR spectra

表 3 预聚体红外羰基区分峰结果

Table 3 Differentiation of $\mathrm{C}=\mathrm{O}$ stretching of different prepolymers in FT-IR spectra

\begin{tabular}{ccccccccc}
\hline \multirow{2}{*}{ Sample } & \multicolumn{3}{c}{$\mathrm{C}=\mathrm{O}$ peak position $/ \mathrm{cm}^{-1}$} & & \multicolumn{3}{c}{$\mathrm{C}=\mathrm{O}$ peak area ratio } \\
\cline { 2 - 3 } & Free & Disordered H-bonded & Ordered H-bonded & & Free & Disordered H-bonded & Ordered H-bonded & \\
\hline $\mathbf{2}$ & 1728 & 1714 & 1694 & & 0.22 & 0.52 & 0.26 & 0.78 \\
$\mathbf{3}$ & 1726 & 1712 & 1691 & & 0.17 & 0.51 & 0.32 & 0.83 \\
\hline
\end{tabular}




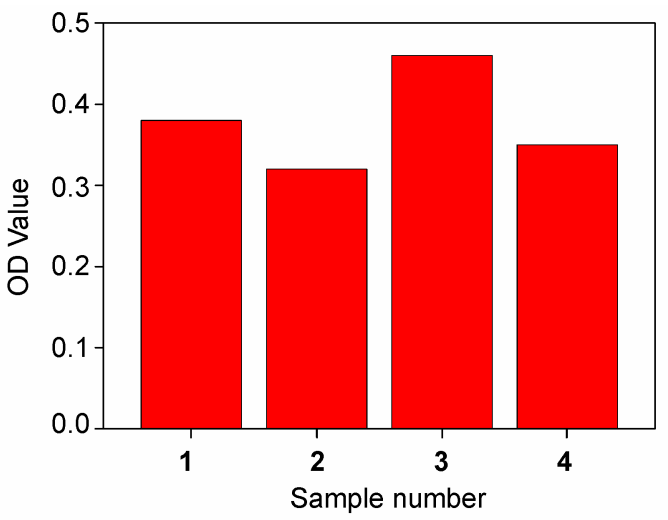

图 5 不同聚氨酯弹性体(样品 1 ～4) 的细胞相容性 Figure 5 Cell compatibility of different polyurethane elastomers

表 4 不同体系聚氨酯的血液相容性

Table 4 Blood compatibility of different polyurethanes

\begin{tabular}{clc}
\hline Sample & Composition & Hemolysis ratio/\% \\
\hline $\mathbf{1}$ & IPDI-PTMG-E300 & 2.8 \\
$\mathbf{2}$ & IPDI-PTMG-BDO & 1.5 \\
$\mathbf{3}$ & IPDI-PC-E300 & 2.1 \\
$\mathbf{4}$ & IPDI-PC-BDO & 0.8 \\
\hline
\end{tabular}

\section{3 实验部分}

\section{1 仪器与试剂}

核磁共振分析仪(Advance 400, 德国 Bruker 公司)、 傅立叶变换红外光谱仪(FT-IR, Nicolet-170X 型, 美国热 电公司)、差示扫描量热仪(Modulated DSC2910, TA 公 司)、力学性能(GMT 系列万能试验机)、接触角测量仪 (Eyma, Japan). 四氢呋喃醚二元醇(PTMG)、聚碳酸酯二 元醇 $(\mathrm{PC})$ 、异佛尔酮二异氧酸酯(IPDI)、1,4-丁二醇 (BDO)和二甲硫基甲苯二胺(E300)均购于上海泰坦科技 股份有限公司.

\section{2 实验方法}

\section{2 .1 材料的合成}

将称量准确的 IPDI 加入到装有搅拌装置、温度传 感器以及干燥高纯氮气导入、导出装置的四口烧瓶中, 外部油浴温度控制在 $60{ }^{\circ} \mathrm{C}$. 缓慢滴加一定量的已经过 高温真空脱水的聚合物二元醇(PTMG, $M_{\mathrm{n}}=2000 ; \mathrm{PC}$, $M_{\mathrm{n}}=2000$ ), 二元醇滴加完毕后再加入预聚物质量分数 为 $0.05 \%$ 的二月桂酸二丁基锡催化剂. 将温度升高并稳 定在 $80{ }^{\circ} \mathrm{C}$ 反应 $3 \mathrm{~h}$, 而后加入准确称量的扩链剂(BDO 或 E300), 边抽真空边慢速搅拌 $2 \mathrm{~min}$, 迅速倒入高温下 涂有脱模剂并预热过的模具中熟化, 得到四种不同种类 的聚氨酯弹性体材料 IPDI-PTMG-E300、IPDIPTMG-BDO、IPDI-PC-E300 和 IPDI-PC-BDO.
IPDI-PTMG-E300: ${ }^{1} \mathrm{H}$ NMR (400 MHz, $\left.\mathrm{CDCl}_{3}\right) \delta$ : $9.10(1 \mathrm{H}, \mathrm{OCONH}), 7.58(2 \mathrm{H}, \mathrm{HNCONH}), 3.41(4 \mathrm{H}$, $\left.\mathrm{OCH}_{2} \mathrm{CH}_{2} \mathrm{CH}_{2} \mathrm{CH}_{2} \mathrm{O}\right), \quad 2.10 \sim 2.60\left(9 \mathrm{H}, \mathrm{SCH}_{3}, \quad \mathrm{NHC}-\right.$ $\left.\mathrm{CCH}_{3}\right), 1.65\left(4 \mathrm{H}, \mathrm{OCH}_{2} \mathrm{CH}_{2} \mathrm{CH}_{2} \mathrm{CH}_{2} \mathrm{O}\right), 1.39\left(2 \mathrm{H},\left[\mathrm{CH}_{3}\right)_{2-}\right.$ $\mathrm{CCH}_{2}$ ], $0.92\left(3 \mathrm{H}, \mathrm{CH}_{3}\right)$; FT-IR (KBr) v: 2973, 2833, 1680, $1385,1253,1109 \mathrm{~cm}^{-1}$. Anal. calcd for C $64.05, \mathrm{H} 9.85, \mathrm{~N}$ 4.51; found C 64.05, H 9.85, N 4.51.

IPDI-PTMG-BDO: ${ }^{1} \mathrm{H}$ NMR (400 MHz, $\left.\mathrm{CDCl}_{3}\right) \delta$ : $9.01(1 \mathrm{H}, \mathrm{OCONH}), 4.02\left(4 \mathrm{H}, \mathrm{OCOCH}_{2} \mathrm{CH}_{2} \mathrm{CH}_{2} \mathrm{CH}_{2} \mathrm{O}-\right.$ $\mathrm{CO}), 3.42\left(4 \mathrm{H}, \mathrm{OCH}_{2} \mathrm{CH}_{2} \mathrm{CH}_{2} \mathrm{CH}_{2} \mathrm{O}\right), 1.65\left(4 \mathrm{H}, \mathrm{OCH}_{2}-\right.$ $\left.\mathrm{CH}_{2} \mathrm{CH}_{2} \mathrm{CH}_{2} \mathrm{O}\right), 1.39$ [2H, $\left.\left(\mathrm{CH}_{3}\right)_{2} \mathrm{CCH}_{2}\right], 0.95\left(3 \mathrm{H}, \mathrm{CH}_{3}\right)$; FT-IR (KBr) $v$ : 2987, 2943, 2821, 1675, 1423, 1303, 1096, $764 \mathrm{~cm}^{-1}$. Anal. calcd for C $65.03, \mathrm{H} 10.28, \mathrm{~N} 3.53$; found C 65.03, H 10.28, N 3.53.

IPDI-PC-E300: ${ }^{1} \mathrm{H}$ NMR (400 MHz, $\left.\mathrm{CDCl}_{3}\right) \delta: 9.15$ $(1 \mathrm{H}, \mathrm{OCONH}), 7.61(2 \mathrm{H}, \mathrm{HNCONH}), 7.09 \sim 7.35(8 \mathrm{H}$, $\mathrm{ArH}), 2.13 \sim 2.60\left(9 \mathrm{H}, \mathrm{SCH}_{3}, \mathrm{NHCCCH}_{3}\right), 1.39[2 \mathrm{H}$, $\left.\left(\mathrm{CH}_{3}\right)_{2} \mathrm{CCH}_{2}\right], 0.90\left(3 \mathrm{H}, \mathrm{CH}_{3}\right)$; FT-IR (KBr) v: 2978, 2843, $1726,1698,1264 \mathrm{~cm}^{-1}$. Anal. calcd for C 69.87, H 6.21, N 4.48; found C 69.87, H 6.21, N 4.48 .

IPDI-PC-BDO: ${ }^{1} \mathrm{H}$ NMR (400 MHz, $\left.\mathrm{CDCl}_{3}\right) \delta: 7.98$ $(1 \mathrm{H}, \mathrm{OCONH}), 7.02 \sim 7.36(8 \mathrm{H}, \mathrm{ArH}), 3.99(4 \mathrm{H}, \mathrm{OCO}-$ $\left.\mathrm{CH}_{2} \mathrm{CH}_{2} \mathrm{CH}_{2} \mathrm{CH}_{2} \mathrm{OCO}\right), 1.69$ (4H, OCOCH $\mathrm{CH}_{2} \mathrm{CH}_{2} \mathrm{CH}_{2-}$ OCO), 1.39 [2H, $\left.\left(\mathrm{CH}_{3}\right)_{2} \mathrm{CCH}_{2}\right], 0.91\left(3 \mathrm{H}, \mathrm{CH}_{3}\right)$; FT-IR (KBr) $v$ : 2954, 2875, 1746, 1443, 1269, 1115, $723 \mathrm{~cm}^{-1}$. Anal. calcd for C 70.72, H 6.71, N 3.51; found C 70.72, H 6.71, N 3.51 .

\subsection{2 傅立叶变换红外光谱(FT-IR)测试}

将 PU 材料制成薄片并将其溶解在四氢呋喃(THF) 中, 配成质量分数为 $2 \%$ 的溶液. 先将溶液均匀涂覆在 盐片上, 并将其置于真空烘箱中除去溶剂. 在红外光谱 仪上进行红外测试, 分辨率为 $4 \mathrm{~cm}^{-1}$. 扫描次数为 16 次, 测定区间为 $500 \sim 4000 \mathrm{~cm}^{-1}$.

\section{2 .3 差示扫描量热(DSC) 测试}

称取 10 15 mg 的 PU 试样, 采用差示扫描量热仪, 在室温至 $250{ }^{\circ} \mathrm{C}$ 范围内, 氮气气氛下进行扫描测试, 升 温速率为 $20{ }^{\circ} \mathrm{C} / \mathrm{min}$.

\subsection{4 材料拉伸强度与断裂伸长率测定}

将 $\mathrm{PU}$ 样条压延成长 $2.0 \mathrm{~cm}$, 宽 $0.5 \mathrm{~cm}$, 厚约为 1 $\mathrm{mm}$ 的薄膜样片. 按 GB1040-92 将薄膜裁切成哑铃型薄 膜样条, 利用万能试验机进行测试, 室温下拉伸速率为 $200 \mathrm{~mm} / \mathrm{min}$.

\section{2 .5 水接触角测试}

将 PU 材料制成薄片并将其溶解在 THF 中，配成浓 度为 $5 \%$ 的溶液, 吸取 1 滴溶液将其滴在载玻片上并快 
速旋转甩干溶剂, 在载玻片上形成一层聚氨酯涂层. 采 用接触角测定仪测定其接触角, 每个样品取三点的平均 值作为测试结果.

\subsection{6 溶血实验}

取新采集人体血液 $20 \mathrm{~mL}$, 加入 $1 \mathrm{~mL}$ 质量分数为 $2 \%$ 的 $\mathrm{K}_{2} \mathrm{C}_{2} \mathrm{O}_{4}$ 溶液制备成新鲜的抗凝血液, 取 $10 \mathrm{~mL}$ 抗 凝血液加入 $10 \mathrm{~mL} 0.9 \% \mathrm{NaCl}$ 溶液进行稀释. 每个配方 下的 PU 材料样品称取 3 份, 每份 $5 \mathrm{~g}$, 分别置于 3 个硅 化试管中(试管硅化方法: 取 $2 \mathrm{~mL}$ 质量分数为 $3 \%$ 的二 氯二甲基硅烷 $\left(\mathrm{Si}\left(\mathrm{CH}_{3}\right)_{2} \mathrm{Cl}_{2}\right)$ 的丙酮溶液涂于试管内壁, 于 $150 \sim 250{ }^{\circ} \mathrm{C}$ 的烘箱中干燥 $6 \sim 8 \mathrm{~h}$, 并加入 $10 \mathrm{~mL}$ 生 理盐水, 随后将硅化试管置于 $37{ }^{\circ} \mathrm{C}$ 恒温水浴箱中, 30 $\min$ 后加入已经稀释的新鲜抗凝血液 $0.5 \mathrm{~mL}$ 并保温 60 $\min$.

阳性对照 3 份: 蒸馏水 $10 \mathrm{~mL}$ 加稀释人体血液 0.2 $\mathrm{mL}$. 阴性对照 3 份: 质量分数为 $0.9 \%$ 的 $\mathrm{NaCl}$ 溶液 10 $\mathrm{mL}$, 加稀释人体血液 $0.2 \mathrm{~mL}$. 将上述所有硅化试管离 心 $5 \mathrm{~min}$ (转速: $1000 \mathrm{r} / \mathrm{min}$ ), 吸取上层清液并移入比色典 内, 采用 721 分光光度计在 $545 \mathrm{~nm}$ 处测定吸光度.

PU 样品的溶血程度按以下公式计算:

溶血率 $/ \%=($ 样品吸光度一阴性吸光度 $) /$

(阳性吸光度一阴性吸光度) $\times 100 \%$

\subsubsection{MTT 法细胞相容性测试 $[22,23]$}

在实验用小鼠脾细胞发生增殖活化时, 细胞内线粒 体琥珀酸脱氢酶活性会升高, MTT [3-(4,5-二甲基-2-噻 唑)-2,5-二苯基溴化四唑]作为底物可参与反应并形成蓝 色的甲臜(Formazan)颗粒, 经盐酸-异丙醇溶解后为蓝色 溶液.

具体实验过程如下: 将合成的聚氨酯弹性体溶于 $\mathrm{CH} 2 \mathrm{Cl} 2$ 中, 并采用旋涂的方式制备尺寸适宜的涂层, 待 $\mathrm{CH} 2 \mathrm{Cl} 2$ 完全挥发, 用乙醇对涂层清洗消毒后放入 96 孔培养板中, 继续使用紫外灯照射 $10 \mathrm{~min}$. 取生长旺盛 的小鼠脾细胞消化、计数, 每孔加入 $100 \mu 1$ 细胞悬液 和 $20 \mu 1 \mathrm{MTT}, 37{ }^{\circ} \mathrm{C}$ 下继续孵化 $4 \mathrm{~h}$. 将每孔上层清液 吸弃, 并继续加入 $150 \mu 1 \mathrm{DMSO}$, 轻微震荡 $20 \mathrm{~min}$, 使结晶物溶解、离心后用酶标测定仪测定细胞培养物的 光吸收值 (OD 值), 测定波长为 $570 \mathrm{~nm}$. 根据 OD 值 的大小计算反应体系中细胞增殖程度
辅助材料(Supporting Information) 聚氨酯弹性体的 红外光谱、核磁共振氢谱谱图. 这些材料可以免费从本 刊网站(http://sioc-journal.cn/)上下载.

\section{References}

[1] Maisonneuve, L.; Lamarzelle, O.; Rix, E.; Grau, E.; Cramail, H. Chem. Rev. 2015, 115, 12407.

[2] Zhang, Y.; Li, Y.; Wang, H.; Zhang, Z.; Feng, Y.; Tian, Q.; Li, N.; Mei, J.; Su, J.; Tian, H. ACS Appl. Mater. Interfaces 2019, 11, 39351.

[3] Huang, Y.; Tang, Z.; Liu, Z.; Wei, J.; Hu, H.; Zhi, C. Nano-Micro Lett. 2018, 10, 38.

[4] Hsieh, C.-T.; Hsu, S.-H. ACS Appl. Mater. Interfaces 2019, 11, 32746.

[5] Mankoci, S.; Ewing, J.; Dalai, P.; Sahai, N.; Barton, H. A.; Joy, A. Biomacromolecules 2019, 20, 4096.

[6] Albers, P. T. M.; van der Ven, L. G. J.; van Benthem, R. A. T. M.; Esteves, A. C. C.; de With, G. Macromolecules 2020, 53, 862.

[7] Li, L.; Chi, X.; Yan, J.; Zhao, Z. Chin. J. Org. Chem. 2018, 38, 955 (in Chinese). (李令东, 迟晓芳, 间佳威, 赵梓含, 有机化学, 2018, 38, 955.)

[8] Best, C. A.; Pepper, V. K.; Ohst, D.; Bodnyk, K.; Heuer, E.; Onwuka, E. A.; King, N.; Strouse, R.; Grischkan, J.; Breuer, C. K.; Johnson, J.; Chiang, T. Int. J. Pediatr. Otorhi. 2018, 104, 155.

[9] Mahanta, A. K.; Mittal, V.; Singh, N.; Dash, D.; Malik, S.; Kumar, M.; Maiti, P. Macromolecules 2015, 48, 2654.

[10] Zhang, Y.; He, X.; Ding, M.; He, W.; Li, J.; Li, J.; Tan, H. Biomacromolecules 2018, 19, 279.

[11] Blakney, A. K.; Simonovsky, F. I.; Suydam, I. T.; Ratner, B. D.; Woodrow, K. A. ACS Biomater. Sci. Eng. 2016, 2, 1595.

[12] Xue, J.; He, M.; Liu, H.; Niu, Y.; Crawford, A.; Coates, P. D.; Chen, D.; Shi, R.; Zhang, L. Biomaterials 2014, 35, 9395.

[13] Zhang, R.-Z.; Ren, Y.-Y.; Yan, D.-K.; Guo, P.-Y.; Li, L.-J. Prog. Org. Coat. 2017, 104, 11.

[14] Xu, Y.; Wang, W.; Chen, J.; Lin, S. Chin. J. Org. Chem. 2018, 38, 2161 (in Chinese). (徐悦莹, 王伟, 陈健壮，林绍梁，有机化学, 2018, 38, 2161.)

[15] Luo, N.; Wang, D.-N.; Ying, S.-K. Polymer 1996, 37, 3577.

[16] Feng, C.; Huang, X. Acc. Chem. Res. 2018, 51, 2314.

[17] Du, J.; Huang, D.; Wei, Y.; Lian, X.; Hu, Y.; Wang, K.; Liu, Y.; Chen, W. Chem. J. Chin. Univ. 2018, 39, 1580 (in Chinese) (杜晶晶, 黄棣, 魏延, 连小洁, 胡银春, 王楷群, 刘阳, 陈维毅, 高等学校化学学报, 2018, 39, 1580.)

[18] Que, Y.; Liu, Y.; Tan, W.; Feng, C.; Shi, P.; Li, Y.; Huang, X. ACS Macro Lett. 2016, 5, 168 .

[19] Gao, A.; Hang, R.; Li, W.; Zhang, W.; Li, P.; Wang, G.; Bai, L.; Yu, X.-F.; Wang, H.; Tong, L.; Chu, P. K. Biomaterials 2017, 140, 201.

[20] Li, X.; Tang, J.; Bao, L.; Chen, L.; Hong, F. Carbohydr. Polym. 2017, 178, 394.

[21] Ma, Z.; Bai, J.; Wang, Y.; Jiang, X. ACS Appl. Mater. Interfaces 2014, 6, 2431.

[22] Zhang, J.; Sun, Z.; Zhu, H.; Guo, Q.; He, C.; Xia, A.; Mo, H.; Huang, X.; Shen, J. J. Mater. Chem. B 2016, 4, 1116.

[23] Nie, S.; Tang, M.; Cheng, C.; Yin, Z.; Wang, L.; Sun, S.; Zhao, C. Biomater. Sci. 2014, 2, 98. 